\title{
Pio II, Pienza e la descrizione di una città intelligente: un'ecfrasi senza retorica
}

\author{
Gabriele Fattorini \\ Università degli Studi di Firenze \\ gabriele.fattorini@unifi.it
}

\section{Riassunto}

Ripercorrendo la lunga e ben nota ecfrasi che Pio II dedicò a Pienza nei Commentarii, il contributo intende evidenziare che Enea Silvio Piccolomini progettò e mise in atto - tramite Bernardo Rossellino - un progetto di città in carne e ossa estremamente razionale, che egli seppe finanziare e costruire mattone per mattone, pietra per pietra, edificio per edificio; una città che innalzò a centro di una nuova diocesi e descrisse senza alcuna retorica. Più che il sogno umanistico di una città "ideale" - come di norma si usa dire - Pienza può dunque apparire come un modello pragmatico e virtuoso di grande attualità, particolarmente a fronte delle elaborazioni troppo spesso fumose e tutte concettuali sul tema della "smart city" che tanta fortuna tendono a riscuotere nella società contemporanea.

Parole chiave: Enea Silvio Piccolomini papa Pio II; Commentarii; Pienza; Rinascimento; architettura; ecfrasi; città ideale; "smart city".

\section{Abstract. Pius II, Pienza and the description of an intelligent city: an ekphrasis with no} rhetoric

The long and well-known ekphrasis dedicated to Pienza by Pius II in his Commentarii testifies how Enea Silvio Piccolomini designed and implemented -through the architect Bernardo Rossellino- an extremely rational project of a new city. A city that he raised to the center of a new diocese and described without any rhetoric. More than the humanistic dream of a "città ideale" -as usually said- Pienza can therefore appear as a pragmatic and virtuous model of great relevance, particularly in the face of the too often smoky and all conceptual elaborations on the theme of the "smart city", that tend to collect so much luck in contemporary society.

Keywords: Enea Silvio Piccolomini Pius II; Pienza; Commentarii; Renaissance; "città ideale"; smart city. 
Pio giunse a Pienza a notte inoltrata; per diversi giorni, costretto a letto dalla malattia, non poté vedere, come avrebbe desiderato, gli edifici che aveva fatto costruire. Non appena stette meglio volle andare a visitarli uno per uno e non si pentì delle spese fatte, benché avesse impegnato in quell'opera più di cinquantamila ducati. La bellezza e la nobiltà dell'edificio gli fecero dimenticare il cruccio della spesa. (Piccolomini, 2008, pp. 1744-1747). ${ }^{1}$

È l'estate del 1462, Enea Silvio Piccolomini ha cinquantasei anni, quasi cinquantasette, e dal 3 settembre 1458 indossa la tiara pontificia; arriva a Pienza ammalato, e certo affaticato dalla lunga carriera diplomatica ed ecclesiastica che ha alle spalle. Una carriera condotta per qualche decennio, calcando le strade di tutta l'Europa e solcando il mare fino alla Gran Bretagna e alla Norvegia, sospinto dalla sua indole pragmatica, da una curiosità inesauribile per gli uomini e le cose, oltre che dal precoce e viscerale amore per le lettere antiche e moderne. Il conclave ha scelto bene: il sottile ingegno, il cospicuo bagaglio culturale e il carattere deciso fanno di Enea una delle personalità di spicco del suo tempo. ${ }^{2}$

La fatica e la malattia passano in secondo ordine, di fronte alla soddisfazione di vedere concretizzato, con rara prontezza, il progetto di trasformare il villaggio natale di Corsignano nella nuova città di Pienza, intitolata al papa e centro di una nuova diocesi, estesa all'interno dell'ambito territoriale dell'antico stato di Siena e inclusa a comprendere, col rango di città, pure la vicina Montalcino. Consapevole che ormai non gli resta troppo da vivere, Enea ha iniziato a raccogliere da qualche mese un resoconto della sua esistenza sotto forma di Commentarii che, soggiunta la morte alla metà d'agosto del 1464, sarebbe rimasto manoscritto nelle mani di Giovanni Gobellino da Lins (Roma, Biblioteca dell'Accademia dei Lincei, Corsiniano 147): più di un secolo dopo, nel 1584, l'arcivescovo di Siena Francesco Bandini Piccolomini, discendente di Enea Silvio, ne avrebbe curato l'editio princeps, dandone una forma assai emendata dalle esigenze della Controriforma, e assegnandone la paternità al Gobellino, col titolo di Pii secundi Potificis Max. Commentarii rerum memorabilium quae temporibus suis contigerunt, a R.D. Ioanne Gobellino vicario Bonnen. Iamdiu compositi a R.P.D. Francisco Bandino Piccolomineo Archiepiscopo senensi ex vetusto originali recogniti et Sanctissimo D.N. Gregorio XIII Pont. Max. Dicati. Come ben noto, scrivendo nell'amato latino, Pio II prese a modello i Commentarii de bello gallico e de bello civili di Cesare, tanto nella scelta di narrare le proprie vicende in terza persona, quanto per l'eccezionale e concreta capacità di sintesi delle descrizioni. Ne esce fuori un racconto biografico esaltante, e uno dei più straordinari spaccati di vita dell'Europa del Quattrocento, del quale non mancano le traduzioni in italiano: in questo caso ci siamo affidati a quella, ormai classica, di Luigi Totaro. ${ }^{3}$

1. Il riferimento è pure alle pagine dell'originale latino a fronte, e così sarà sempre nelle successive citazioni dei Commentarii.

2. Oltre ai Commentarii (e all'introduzione di Luigi Totaro, in Piccolomini, 2008, pp. IXXXXI), rimando a Pellegrini 2000 e 2015, pure per la corposa bibliografia.

3. Per la storia dei Commentarii e le varie edizioni, rimando all'introduzione di Totaro, in 
Nei Commentarii Enea Silvio si sofferma più volte su Pienza, finendo per consacrarle nel nono libro una descrizione assai dettagliata, che prende avvio con il passo trascritto in apertura di questo contributo, e rappresenta uno degli esempi più emblematici della letteratura ecfrastica del Quattrocento: le parole del papa, infatti, non riguardano una serie di opere concepite da altri, ma illustrano con eccezionale meticolosità, e con qualche strepitosa metafora, il risultato del suo mecenatismo. Seguendo il suo discorso, come è stato ben inteso dagli studi, è facile comprendere anche il ruolo decisivo che Pio II ebbe nella progettazione della nuova città, a fianco dell'architetto fiorentino Bernardo Rossellino, e soprattutto il temperamento pragmatico che fu dietro l'intera operazione.

Ciò nonostante Pienza evoca sovente temi come quello della città ideale o della realizzazione di una concettuale "visione umanistica del mondo" (Pieper, 2000). ${ }^{4}$ Se il linguaggio asciutto e rigoroso dei Commentariii è sufficiente a sgombrare il campo da qualsiasi equivoco su ogni astrazione e trasfigurazione, la questione della città ideale - una definizione che non mi risulta esistere nella letteratura del Quattrocento - marcia su un terreno ambiguo, che tende ad alternare la razionalità di un piano urbanistico con l'ambizione di realizzare progetti utopici e i paragoni con celebri immagini dipinte per dimostrare le potenzialità illusionistiche della prospettiva. Non v'è dubbio che la scenografica piazza pientina, quando è fotografata come uno spazio privo di presenze umane, si presti assai bene al confronto con la così detta Città ideale della Galleria Nazionale delle Marche di Urbino, e la ragione è che entrambe sono prodotti della più tipica cultura prospettica quattrocentesca (figg. 2-3). ${ }^{5}$ Tuttavia c'è da stare certi che Pio II quando pensava alla sua piazza - per la quale il Rossellino aveva escogitato una celeberrima pianta trapezoidale, utile a farla apparire illusionisticamente più grande, e ad aprire vie di fuga ai lati della Cattedrale, verso il paesaggio dell'Amiata - non la immaginava vuota, ma piena di gente, come l'aveva vista il 21 settembre 1462, per la fiera di San Matteo. Allora aveva assistito "non senza letizia" e "insieme con i cardinali" alle corse di cavalli, asini, uomini e fanciulli che rallegrarono la città, affacciandosi "da una finestra altissima" del suo palazzo, e "non trascurando però nel frattempo di trattare gli affari di stato" (Piccolomini, 2008, pp. 1776-1777). Una dichiarazione di scrupolosa dedizione al lavoro che dice molto della personalità del pontefice.

\section{Piccolomini, 2008, pp. XXXV-LIII.}

4. Su Pienza la letteratura è ormai sterminata, ma un imprescindibile punto di partenza resta ancora Mannucci, 1937; in seguito segnalo almeno: Carli, 1966; Mack 1987; Tönnesmann, 1990; Adams 1998; Pieper, 2000 (dove le letture risultano troppo spesso arbitrarie e distanti dal pragmatico temperamento di Pio II); Mack, 2012; Spesso, 2018 (pure con passaggi fin troppo concettosi).

5. Va sottolineato che il titolo di Città ideale è stato assegnato alla tavola di Urbino (e a quelle del Walters Art Museum di Baltimora e della Gemäldegalerie di Berlino) solo in tempi relativamente recenti; non sappiamo come definissero queste immagini coloro che le commissionarono, ma possiamo essere certi che si tratta di esercizi prospettici, che meriterebbe definire più correttamente "prospettive urbane" (Angelini, 2011-2012). 
D'altronde anche la vicenda dell'origine di Pienza fu un affare di stato, peraltro fuori del comune. Nessuno prima di Enea Silvio Piccolomini, infatti, aveva dato vita a una nuova diocesi per ragioni estranee a fini pastorali, e per realizzare i suoi propositi il pontefice aveva agito con machiavellica decisione:

Pio eresse Montalcino in città, a pari grado con Pienza, e in entrambe le città istituì una chiesa episcopale e un capitolo di canonici; ma volle che uno solo fosse il vescovo e una la diocesi; questa venne costituita in parte con parrocchie dei vescovi di Chiusi e di Arezzo, e in parte con villaggi e possedimenti che [...] erano soggetti al monastero di Sant'Antimo. Pio convinse l'abate a cedere quei suoi diritti, offrendogli in cambio un vitalizio; incorporò quindi il monastero tra i privilegi del nuovo vescovo e fornì il denaro necessario per riscattare $\mathrm{i}$ terreni venduti e coltivarli. (Piccolomini, 2008, pp. 1578-1579).

Dietro questa abile, inedita, arbitraria e scaltra operazione giuridica, Giuseppe Chironi ha ben individuato, nella singolare anomalia istituzionale dell'origine di Pienza, la precisa volontà politica di Enea Silvio Piccolomini di creare un "mondo perfetto". ${ }^{6}$ Fin da quando era stato incoronato pontefice, Pio II aveva infatti cercato in tutti i modi di intervenire nella politica interna del Comune - alias Repubblica - di Siena, incoraggiando riforme mirate a risolvere l'atavico problema delle fazioni che affliggeva la politica senese: una questione che egli aveva a cuore perché toccava anche la sua famiglia, che il proverbiale temperamento nepotistico del pontefice cercò sempre di favorire e allargare. Su questo fronte, però, non ci fu nulla da fare, e una volta preso atto dell'impossibilità di sanare la civitas senese, il pontefice, in virtù della propria autorità, finì per "costruirsi una città su misura in cui esercitare i propositi di ingegneria sociale e politica, in cui ricreare quella concordia ordinum, quella solidarietà dei ceti dominanti che costituiva un modello ideale di convivenza civile, ormai impossibile a Siena" (Chironi, 2009, p. 42). Un tale progetto prese avvio verso la metà di febbraio del 1462 e si realizzò pochi mesi dopo, quando il 29 agosto 1462 Pio II consacrò la Cattedrale di Pienza e nominò vescovo Giovanni Cinughi, "dando così uno sposo alla sposa novella" ("et novae sponsae virum dedit"; Piccolomini, 2008, pp. 1770-1771).

I tempi poterono essere brevissimi, perché a Corsignano erano stati avviati i due grandi cantieri della chiesa (poi Cattedrale) e della dimora del pontefice (il Palazzo Piccolomini) fin dal 1459. Allora, tuttavia, si trattava di una questione privata, di lasciare memoria di sé attraverso interventi significativi e mirati, un po' come aveva fatto un quarto di secolo prima il cardinale Branda Castiglioni nel borgo di famiglia di Castiglione Olona, in Lombardia. ${ }^{7}$ Tutto ciò aveva avuto origine nel febbraio del 1459, quando Enea Silvio era tornato per la prima volta da papa nell'amato villaggio natio, tratteggiandone nei Commentarii uno scorcio quasi a volo d'uccello (fig. 1):

6. Chironi, 2009: una voce bibliografica chiave per la costruzione giuridica di Pienza. Per la bolla del 13 agosto 1462, con la quale fu istituita la diocesi: Chironi, 2000, pp. 18. 515-518 n. 2.

7. Sul caso di Castiglione Olona: Spiriti, 2018. 
C'è un colle che s'innalza nella valle del fiume Orcia, sulla cui cima si apre un pianoro lungo mille passi e largo molto meno. Su un angolo del pianoro, nella parte che d'inverno guarda verso il sole nascente, sta un villaggio non molto conosciuto, ma con un'aria salubre, ottimo vino e tante buone cose da mangiare. Coloro che da Siena vanno a Roma, dopo aver passato il castello di San Quirico, proseguendo alla destra di Radicofani, passano accanto a Corsignano, che si vede in alto sul colle, alla sinistra, in cima a un dolce pendio, a tre miglia dalla via principale. (Piccolomini, 2008, pp. 312-313).

Pare di leggere una guida turistica dei nostri giorni, di quelle scritte bene e con amore, tanto Enea Silvio ha la capacità di enucleare le specificità di un luogo che non sempre ha goduto di fama: infatti la Val d'Orcia, tanto osannata dal turismo "lento" dei nostri giorni, in passato fu sovente giudicata dai viaggiatori come un noioso e solitario segmento della lunga strada tra Siena e l'Urbe. ${ }^{8}$

$\mathrm{Al}$ di là dell'amena atmosfera dell'ecfrasi, l'impatto con Corsignano finisce per essere pieno di malinconia, perché

Egli sperava di trovare un qualche piacere a parlare con coloro che erano cresciuti insieme con lui, e sperava di rivedere con gioia il suolo natale. Ma avvenne il contrario, poiché gran parte dei suoi compagni era morta e coloro che ancora vivevano erano trattenuti in casa dal peso degli anni e delle malattie; e se alcuni si facevan vedere, a stento si potevan riconoscere, tanto i loro volti erano mutati, e tanto erano indeboliti e storpiati e quasi messaggeri di morte. Dovunque il papa rivolgeva lo sguardo, scorgeva i segni della sua vecchiezza, e non poteva non ammettere d'essere vecchio e vicino a morire, poi che aveva trovato ormai carichi d'anni i figli di coloro che aveva lasciato fanciulli. (Piccolomini, 2008, pp. 312-313).

Acclamato con gioia dai Corsignanesi, il papa celebrò la messa il 22 febbraio, per la festa della cattedra di San Pietro, e poi "decise anche di costruire in quel luogo una nuova chiesa e un palazzo, per questo assunse con non piccola spesa architetti e muratori, onde lasciare un ricordo duraturo della sua origine" (Piccolomini, 2008, pp. 314-315). Al tempo edace, che aveva rattristato il ritorno in patria, Enea Silvio contrappose prontamente un magnificente progetto di ars aedificatoria, pensato per celebrare il suo nome e la sua schiatta nei secoli, e i cantieri furono approntati con una rapidità impensabile per i nostri giorni, tanto che nel settembre successivo - quando trascorse un breve e ulteriore soggiorno a Corsignano, afflitto inizialmente dalla malattia - il "papa vide con il più grande piacere che stavano sorgendo sul suolo natio degli edifici non inferiori a nessun altro in Italia" (Piccolomini, 2008, pp. 784-785). ${ }^{9} \mathrm{E}$

8. Emblematico il caustico giudizio del francese Charles de Brosses (1973, p. 231) sul Palazzo Zondadari del vicino borgo di San Quirico d'Orcia: "una casa così bella in un luogo tanto brutto". A pochi chilometri di distanza, Pienza restava appena al di fuori della strada per Roma, e quindi estranea al passaggio dei viaggiatori del tempo del Grand tour.

9. Nella traduzione di Totaro mi sono permesso di sostituire "edifici" a "palazzi", perché il cantiere ebbe il suo cardine nella Cattedrale, oltre che nel Palazzo Piccolomini. Per i tempi del cantiere pientino, e il suo carattere inizialmente privato: Adams, 1985, Mack, 1987, pp. 43-155; Adams, 1998; Mussolin, 2005, pp. 230-235; Mack, 2012. 
lo stesso Pio II a dirci con orgoglio, in un passo del nono libro dove descrive prima il suo palazzo e poi la Cattedrale, che "tutto ciò venne condotto a termine, dalle fondamenta sino al tetto, nel giro di tre anni, con l'eccezione del campanile [della Cattedrale] che non era ancora finito" (Piccolomini, 2008, pp. 1764, 1766-1767).

Chiunque visiti Pienza con una qualche consapevolezza può agevolmente rendersi conto che è difficile - se non impossibile - rappresentarla con parole migliori di quelle che le dedicò colui che, al tempo stesso, fu il suo padre e il figlio più illustre di Corsignano. Partiamo allora dalla dimora del papa (fig. 4):

Il palazzo, quadrato, era alto novanta piedi, costruito dalla base sino in cima in bozze di pietra tagliate a regola d'arte e con gli spigoli incassati per la profondità di un dito, in modo che le giunture combaciassero perfettamente $\mathrm{e}$ le sporgenze delle pietre risaltassero a guisa di tessere. Sul tetto c'era un compluvio costruito con pietre più grandi, rientrante di cinque piedi dal muro, che raccoglieva le acque piovane nei canali del tetto e le portava lontano per mezzo di tubi di ferro. Lo spessore del muro in nessun punto era inferiore a sei o quattro piedi. Tre lati del palazzo erano costruiti in questo modo, e di questi il lato a settentrione era lungo centoventisei piedi, quelli a oriente e occidente erano più lunghi di diciotto piedi per la presenza dei porticati che sul lato a mezzogiorno sporgevano rispetto al corpo quadrato del palazzo. Il perimetro dell'intero edificio era di 540 piedi.

C'erano due ordini di finestre ammirevoli per forma e ampiezza, e ciascun ordine era formato da ventritre finestre equidistanti fra loro. Da ognuna delle finestre, divise da colonnette, potevano contemporaneamente affacciarsi tre persone. Sotto a tutt'e due gli ordini di finestre correvano, attorno al palazzo, cingendolo come corone, due cimase ornamentali, dette comunemente cornici, fatte di travertino e splendidamente lavorate. L'architetto innalzò anche da terra sino al compluvio delle lesene quadrate armoniosamente inserite, con basi e capitelli molto appropriati. Negli angoli del palazzo, e in più punti tra una finestra e l'altra, appese scudi di pietra sui quali risplendevano in oro, argento e altri colori le armi della casa Piccolomini, realizzate con l'arte dello scultore e insieme del pittore. Erano stati collocati anche molti anelli di ferro e bracci per reggere fiaccole accese durante la notte o porvi bandiere quando fosse stato necessario. C'erano inoltre finestre più piccole di forma quadrata, che davano luce alle stanze del pianterreno, e queste erano chiuse con grate di ferro. Non mancavano infine tutt'attorno al palazzo sedili di due gradini e in certi punti anche di tre gradini, fatti della stessa pietra delle cornici. (Piccolomini, 2008, pp. 1744, 1747-1749).

È un'ecfrasi perfetta, di straordinaria lucidità, che accenna pure a certi dettagli che non sono arrivati fino a noi, come l'originaria policromia delle armi piccolominee in facciata; nell'indugiare fin dal principio sulle misure, sui materiali e addirittura su quelli che oggi chiameremmo gli impianti, pare quasi la relazione di un tecnico. Mancano invece quei dati che troviamo in ogni buon manuale di storia dell'arte o dell'architettura, quanto al fatto che il Rossellino avesse progettato l'edificio guardando per il prospetto a bugnato al Palazzo Rucellai di Firenze, nato da un disegno di Leon Battista Alberti e costruito 
sotto la direzione del medesimo Bernardo dagli anni Cinquanta, ma con tempi assai dilatati (tanto che dovette essere terminato dopo Palazzo Piccolomini). La pianta quadrata, organizzata intorno a un cortile che conduce a un giardino, rimanda invece alla grandiosa dimora di Cosimo il Vecchio, ultimata sotto la supervisione di Michelozzo intorno al 1459-1460, e che il Piccolomini (2008, pp. 352-353) ebbe a giudicare un "palacium rege dignum", cioè "degno di un re". ${ }^{10}$ Nell'ampia e dettagliata immagine del Palazzo Piccolomini riferita dal pontefice mai si accenna a tali discendenze fiorentine, né è uno sguardo ai moderni elementi architettonici che associamo al linguaggio rinascimentale: dall'utilizzo dell'arco a tutto sesto per le bifore, alla scelta di differenziare la tipologia dei peducci a ogni piano, in nome della sovrapposizione degli ordini con una nuova e corretta successione teorizzata da Vitruvio, testimoniata dal Colosseo e rilanciata da Alberti teorizzata da Vitruvio, testimoniata dal Colosseo e rilanciata da Alberti. ${ }^{11}$

Ciò non toglie che Pienza rappresenti un chiaro esempio della diffusione di quella cultura dell'antico in senso cristiano con la quale Pio II aveva familiarizzato nella Roma dell'amico Niccolò V, e sulla quale aleggiava la figura di Leon Battista Alberti, in cui furono avviati progetti ambiziosi, come la ristrutturazione della Basilica Costantiniana, che vide peraltro il coinvolgimento del Rossellino. ${ }^{12}$

Per ragioni di brevità non seguiremo Pio II nel prolungato e puntale tour della propria dimora. È sufficiente limitarsi a selezionare qualche brano, utile per esempio a cogliere il suo interesse per la tecnologia, come quando elogia il sistema di raccolta delle acque piovane, che dal tetto, tramite apposite canalizzazioni, finivano in un paio di ampie cisterne, rispettivamente nel palazzo e nel giardino. Tecnologia che non andava disgiunta dalle esigenze estetiche, tanto che i ventitré comignoli sul tetto erano ornati con pinnacoli dipinti, perché da lontano aggiungessero "molto splendore e bellezza all'edificio" (Piccolomini, 2008, pp. 1748-1751). Fondamentale il valore che egli dava ai comfort, uniti alle finiture di pregio:

tutte le stanze da letto erano provviste di camino e di ogni altra struttura necessaria, ovunque erano soffitti a cassettoni degni d'essere visti: le travi d'abete di grandezza e bellezza appropriata al palazzo, i travetti e i pannelli di legno conferivano una straordinaria ricchezza sia con la loro forma, sia perché dipinti o ricoperti con lamine d'oro. I pavimenti erano di mattoni accuratamente levigati, perfettamente uniformi, tutti uguali: in nessun punto c'era uno scalino da salire o discendere per passare da una stanza all'altra o da uno ad un altro luogo. (Piccolomini, 2008, pp. 1752-1755). ${ }^{13}$

10. Il passo è citato peraltro da Nevola (2005, pp. 184-189), cui rimando - con bibliografia pure per i caratteri del palazzo.

11. Ibidem, ma la questione è ben nota.

12. Su questo ambiente complesso e il suo rilievo per la formazione dei gusti di Pio II, mi limito a ricordare: Angelini, 2005, pp. 19-21 e Mussolin, 2005, pp. 215-227.

13. Subito sopra, quanto a comfort, il Piccolomini (2008, pp 1754-1755) accenna che per la sua camera da letto "aveva dato ordine di rivestire le pareti d'abete per evitare che l'umidità 
La qualità principale del palazzo era tuttavia la luminosità degli ambienti, e di ciò il papa ne fa giustamente un vanto, muovendo da un assunto quanto mai attuale:

E se, come alcuni pensano, il principale pregio di una casa è la luce, allora certamente nessuna dimora potrà esser preferita a questa, che si apre senza che nulla si interponga verso i quattro punti cardinali e fa entrare abbondante la luce non solo dalle finestre esterne, ma anche da quelle che danno sul cortile interno del palazzo, distribuendola così fino ai recessi più bassi. (Piccolomini, 2008, pp. 1754-1755).

E il tema della luce - come ben noto - si lega a quello del paesaggio, poiché le finestre si aprono su splendidi panorami, oltre modo apprezzati dal Piccolomini (2008, pp. 1754-1757): verso occidente lo sguardo si spinge sino a "Montalcino, e a Siena e alle stesse montagne pistoiesi"; a nord vi sono colli e boschi per cinque miglia, e chi ha gli occhi buoni può vedere l'Appennino e "Cortona alta su un colle". A oriente la visuale "è più ristretta e non va oltre Montepulciano e i monti che dividono la Val di Chiana dalla Val d'Orcia. Questa, con i suoi "prati verdeggianti e i colli nella stagione propizia coperti d'erba, e i campi fruttiferi e le vigne, e su rupi scoscese, le rocche e i borghi, e i bagni detti di Vignone e il Monte Pesio, che è sopra Radicofani, porta del sole invernale", si distende a meridione, all'orizzonte di quanto si ammira dalle tre logge, e al di sotto del possente e boscoso Monte Amiata, l'amato luogo di villeggiatura del pontefice (fig. 5). ${ }^{14}$

$\mathrm{E}$ un affaccio che ci lascia senza fiato in ogni stagione dell'anno, così come deve avere ammaliato gli ospiti che nei secoli hanno avuto la fortuna di essere accolti nel triplice loggiato affacciato sulla Val d'Orcia. Accanto a questo, sul fianco occidentale, sorge una curiosa struttura adiacente e collegata al corpo del palazzo, che il Piccolomini (2008, pp. 1756-1757) spiega orgogliosamente essere stata studiata per accogliere cucine capaci di attingere acqua dalla sottostante cisterna e servire tutti i piani delle dimora. Nessuno tuttavia vi fa caso, e anche questo è un pregio, perché l'attenzione è semmai attratta dall'ultimo elemento del palazzo che resta da descrivere: un elegante giardino collegato con il cortile del piano terra, e che in realtà è un giardino pensile. Pio II spiega

dei muri freschi nuocesse". Pur senza gli arredi originali, quest'ultima occupa ancora lo spazio in cui la menzionava Enea Silvio nel suo racconto del piano nobile si veda: Martini, Santi, 2006, pp. 53-57 (nonché il resto della guida per una accurata descrizione degli interni del palazzo nelle forme attuali).

14. Per ricordare la passione di Pio II per il paesaggio (in ultimo Spesso, 2018, pp. 66-69) basta avere a mente la definizione di "silvarum amator" (nonché "varia vivendi cupidus") che il Piccolomini (2008, p. 1652) dette di sé stesso, tra le righe del primo capitolo del nono libro dei Commentarii, reso famoso da un'idilliaca vacanza al Monte Amiata contraddistinta da una totale immersione nel verde della natura, che già Jacob Burckhardt (1876, II, pp. 32-36, in particolare pp. 35-36) segnalò come emblematica testimonianza rinascimentale della "scoperta del bello nel paesaggio". Del tema della luce, che come vedremo unisce Palazzo e Cattedrale, ha detto in ultimo, nel contesto della sua fortuna umanistica, Mario Bevilacqua (2016, p. 34). 
con accuratezza che il giardino è stato costruito su un "terreno ineguale e in forte pendenza", e che al di sotto si trovano le stalle e le botteghe dei fabbri, ospitate nella struttura necessaria affinché uno "spiazzo grande quanto quello occupato dal palazzo", esteso fino alle mura della città, potesse essere spianato. Un giardino "adatto a piantarvi viti e alberi", completato tutto intorno con sedili in pietra e chiuso da un elegante parapetto e collegato con il resto.

Chi entra nel palazzo per la porta principale dal lato di settentrione raccoglie in un solo sguardo il peristilio e il cortile e, al di là della porta posteriore, il loggiato e il giardino in tutta la sua lunghezza, e può passeggiare tranquillamente per tutto questo spazio senza mai dover superare un gradino. (Piccolomini, 2008, pp. 1756-1757).

A parte la battuta su quest'ultima comodità - tanto opportuna per un padrone di casa che, per la malattia, poteva avere difficoltà a deambulare, e nelle sue descrizioni rivela dunque una vera mania per contare il numero dei gradini in queste righe che chiudono la lunga descrizione del palazzo, il papa mette chiaramente in parole la spettacolare fuga prospettica che il Rossellino aveva concepito per impressionare gli illustri e colti ospiti del suo committente (fig. 6). Un'ecfrasi che infatti si può tranquillamente traslare a quella pittura prospettica che aveva a monte le inclinazioni dichiarate da Leon Battista Alberti nel De pictura e che ebbe tra i suoi campioni Domenico Veneziano e il suo allievo Piero della Francesca, trovando terra fertile, al tempo di Pio II, anche nella pala che il senese Lorenzo di Pietro detto il Vecchietta dipinse per la grancia di Spedaletto nei pressi di Pienza (ora nel Museo Diocesano; fig. 7). Una pittura "in cui i colori si imperlano di luce e la prospettiva diventa uno spettacolo per gli occhi" (Bellosi, 1990, pp. 11-12). ${ }^{15}$

Non minore è l'attenzione riservata dal Piccolomini (2008, pp. 17581759), nel successivo capitolo del nono libro, al "tempio in onore della beata Maria sempre vergine" costruito accanto al palazzo, spiegando innanzitutto che "la forte pendenza del terreno fece sì che esso fosse costituito di due chiese, una superiore e una inferiore". Il cantiere, per necessità, era sorto su di un'area geologicamente poco adatta, alcuni operai erano morti "precipitando per il dirupo roccioso, che non era sufficientemente protetto" (e qui si riconosce un accenno alla questione della sicurezza, oggetto ai nostri giorni di un'attenzione assai maggiore), e "una crepa apparsa nell'edificio, dalla base alla cima, fa nascere qualche sospetto sulla saldezza delle fondamenta". Alle rassicurazioni dell'architetto, "che ritenne che non si dovesse temere per la struttura dell'edificio", il papa rispose con filosofia: "il tempo mostrerà se è vero". E il tempo ha dato ragione al Rossellino, perché la chiesa è ancora in piedi, anche

15. È una citazione che ho richiamato a proposito dei valori che percorrono la pala di Spedaletto del Vecchietta (Fattorini, 2006), di cui illustro il luminoso loggiato chiuso da un hortus conclusus dell'Annunciazione: tema caro pure a Domenico Veneziano e Piero della Francesca, si vedano rispettivamente la tavoletta del Fitzwilliam Museum di Cambridge per la predella della pala di Santa Lucia de' Magnoli a Firenze e il coronamento del polittico della Galleria Nazionale dell'Umbria di Perugia. 
se le grandi crepe sulle pareti e sul pavimento, proprio nella zona di confine tra le navate e il presbiterio, costringono a continui monitoraggi e periodici interventi di restauro. ${ }^{16}$

La visita inizia con la chiesa inferiore - cioè il Battistero (così com'è a Siena) - e dopo la solita ossessione per il conteggio dei gradini per i quali vi si scende (sedici) e il ricordo dei possenti pilastri centrali posti a sostenere l'intero edificio, il papa indica immediatamente quell'elemento chiave dell'architettura sacra pientina, che l'accomuna alla principale peculiarità del palazzo: "la luce, che entra abbondantemente da tre grandi finestre, illumina tutta la chiesa e i quattro altari e il fonte battesimale, che è costruito con una pietra bianca con eccellente lavoro in una delle cappelle del tempio". ${ }^{17}$ In conseguenza di ciò "l'aspetto della chiesa suscita in colui che entra un sentimento di commozione e di profonda, religiosa devozione" (Piccolomini, 2008, pp. 1758-1759, 1761).

Un obiettivo che Enea Silvio e il Rossellino - committente e architetto - realizzarono ancora meglio nella soprastante Cattedrale, della quale si indicano in principio le misure (centoquaranta piedi di lunghezza, sessanta piedi di altezza e pure di larghezza, senza contare le cappelle) e si giustifica l'orientamento "da settentrione verso mezzogiorno" per "ragioni di necessità", connesse ovviamente con l'impianto urbano della città e la preesistenza della precedente chiesa parrocchiale (Piccolomini, 2008, pp. 1760-1761). ${ }^{18}$ Della facciata il pontefice mette in evidenza il candore della pietra e quegli elementi che riconduciamo al linguaggio rinascimentale, ovvero il carattere antiquario - "presenta l'aspetto dei templi antichi, magnificamente adorna di colonne, archi e nicchie che possono accogliere delle statue" - e i proporzionati ed eleganti elementi decorativi:

$\mathrm{Ci}$ sono tre porte armoniosamente proporzionate, quella di centro più ampia delle altre, e un grande rosone, che sembra l'occhio di un ciclope; ci sono quindi lo stemma dei Piccolomini e sopra questo la tiara papale cinta dalle tre corone e, fra lo stemma e la tiara, le chiavi della Chiesa. La facciata dalla base sino al tetto mantiene sempre la stessa larghezza, per terminare poi in forma di piramide, cinta da belle cornici. (Piccolomini, 2008, pp. 1760-1761).

Viene quindi il momento di accedere al tempio, e di un passo assai celebre, perché testimonia bene un effetto comune a ogni novizio che per la prima

16. Il restauro fondamentale rimane quello compiuto nella prima metà degli anni Trenta del Novecento, che non si occupò solo del consolidamento, ma dette alla Cattedrale l'aspetto attuale, eliminando le "aggiunte" di età moderna, come spiegava Alfredo Barbacci (1934), che progettò e diresse i lavori. Per il recente monitoraggio: Bulian, Croci, Giorgianni $\&$ Russo, 2006. Mentre sto scrivendo è in via di smontaggio il cantiere di un recentissimo restauro.

17. Rispetto alla traduzione di Totaro mi sono permesso di sostituire "marmo" con "pietra bianca”, perché il fonte battesimale è realizzato in travertino.

18. Per un'acuta lettura della Cattedrale di Pienza, nel suo contesto storico e culturale, con ripetuti riferimenti ai Commentarii e ovviamente una ricca bibliografia, rimando a Mussolin, 2005. Le tracce della precedente parrocchiale di Santa Maria furono rinvenute da Barbacci (1934, pp. 9, 118, 120). 
volta sperimenta la visione di questo spazio: "chi entra per la porta centrale si trova davanti agli occhi tutta insieme la chiesa, con le sue cappelle e gli altari, mirabili per la straordinaria luminosità e per lo splendore dell'architettura" (Piccolomini, 2008, pp. 1760-1761, 1763). Tale effetto è conseguenza di una precisa scelta del papa, ancor più che dell'architetto: subito dopo egli descrive infatti la struttura ad Hallenkirche - "tre navate di cui quella centrale è più ampia, mentre in altezza si eguagliano" -, tenendo a puntualizzare che "così era stato disposto da Pio, che aveva preso il modello presso i popoli tedeschi in Austria", e che "una simile disposizione rende la chiesa più bella e più luminosa" (Piccolomini, 2008, pp. 1762-1763). Tra i modelli chiamati in causa, quello che pare funzionare meglio è la cappella palatina di San Giorgio entro il castello di Wiener Neustadt, che Enea Silvio ebbe modo di frequentare quando fu al servizio dell'imperatore Federico III. ${ }^{19}$

Mirabile è l'epifania dell'abside, che per essere innalzata su di una pianta semicircolare, appare "come un capo incoronato", perché "è divisa in cinque cappelle che si protendono in fuori rispetto al resto della struttura, ed ha altrettante volte, uguali alle navate in altezza, che per le stelle d'oro su di esse applicate e il colore dorato di cui sono dipinte imitano l'aspetto verace del cielo" (Piccolomini, 2008, pp. 1762-1763). Delle varie cappelle dell'abside sono ben definite le funzioni: quella centrale accoglie un coro intagliato in legno e intarsiato, con la cattedra episcopale e gli stalli dei canonici, nella cappella accanto a sinistra (guardando verso l'ingresso della chiesa) è un monumentale tabernacolo del sacramento in coro intagliato e intarsiato, mentre nelle altre ci sono altari "adorni di tavole dipinte, opere di illustri pittori senesi" (Piccolomini, 2008, pp. 1762-1765). Pio II non fa i nomi di Lorenzo di Pietro detto il Vecchietta, Matteo di Giovanni, e Sano di Pietro, che pure doveva conoscere benissimo per averli ingaggiati e pagati, e non si sofferma sul fatto che nel suo Duomo compaiono le prime pale della pittura senese che adottano consapevolmente un formato rinascimentale (certo perché disegnato dal medesimo Rossellino per uniformarsi allo spazio da lui studiato), pur mantenendo il tradizionale fondo dorato. ${ }^{20}$ Molto più gli interessa tornare sulla questione della luce, e al fatto che "tutte le cappelle hanno una finestra ampia e alta, elegantemente adornata di esili colonne e fioroni di pietra, chiusa con vetro detto cristallino. $\mathrm{Ci}$ sono anche altre quattro finestre uguali nelle navate laterali, e attraverso di esse quando splende il sole entra tanta luce che chi si trova nel tempio pensa di trovarsi racchiuso non in una casa di pietra, ma di vetro" (Piccolomini, 2008, pp. 1764-1765). È un passaggio cruciale, che dichiara la specificità del tempio pientino, e lascia intendere la discendenza dall'impatto che certi edifici sacri gotici avevano avuto sul giovane Enea Silvio, e particolarmente, in terra d'Inghilterra, la Cattedrale di York (figg. 8-9):

19. Spesso 1997; Mussolin, 2005, pp. 242-243, 239 (con riferimento ad alcuni tra i più significativi interventi sul tema dell'Hallenkirche pientina).

20. Sulle pale del Duomo di Pienza: Van Os, 1987; Palladino, 1994; Martini 2005; Martini 2009; Paardekooper 2009, pp. 377-394 (di cui condivido le conclusioni sulla discussa questione della collocazione degli altari). 
"famosa in tutto il mondo per la sua vastità e la bellezza della costruzione, e per una cappella assai luminosa che ha pareti di vetro sostenute da esilissime colonne" (Piccolomini, 2008, pp. 28-29). ${ }^{21}$

Alla volontà di fabbricare una casa di luce, in cui non vi fossero schermi tra le finestre e lo spazio della liturgia, si lega evidentemente la scelta, contraria alla consuetudine medievale, di evitare uno spesso tramezzo tra il clero e i fedeli, e di avere così un altare maggiore - "collocato fra le due ultime colonne" e sopraelevato su "quattro gradini" - senza pala d'altare e senza tabernacolo eucaristico; di conseguenza "il sacerdote e i suoi ministri quando celebrano la messa hanno dietro a sé il popolo e davanti a sé i cantori, di fronte alla cattedra pontificale" (Piccolomini, 2008, pp. 1764-1765). ${ }^{22}$

Il pontefice non manca di ricordarci che la Cattedrale era fornita di due belle acquasantiere in prossimità dell'ingresso, di due altari "a disposizione dei fedeli" nella prima parte dell'edificio, dei quali non sono arrivate a noi le mense, ma solo le pale compiute dopo la consacrazione, nel 1463 (quella di Giovanni di Paolo) e verso il 1464 (l'altra di Matteo di Giovanni), della sagrestia ubicata sulla destra, della "torre campanaria alta centosessanta piedi, della quale al presente manca un terzo" (e che poi sarebbe stata completata), e di due scale a chiocciola nascoste nelle murature per salire "dalla chiesa inferiore alla superiore sino al tetto". ${ }^{23}$

Il Piccolomini (2008, pp. 1764-1765, 1767) conclude quindi il tour del Duomo tornando nella piazza antistante, per soffermarsi sul "pozzo profondo di acqua viva, la cui bocca fu adornata di marmi, con due colonne che sostengono una trave di marmo elegantemente scolpita e le catene e i secchi necessari per cavare l'acqua": dunque non fu pensato come semplice elemento di arredo urbano (come tende ad apparire oggi), ma con una funzione assai pratica.

$\mathrm{Al}$ responsabile di tutto questo, Bernardo Rossellino, Enea Silvio dedica il capitolo successivo, raccontando l'aneddoto di come il papa, contro le invidie e le malelingue che accusavano l'architetto di avere presentato un preventivo assai minore rispetto alle spese finali, volle pagarlo cento ducati in più del pattuito, affidandogli nuove commissioni, e ringraziandolo con queste parole:

Bene hai fatto, o Bernardo, dicendoci il falso sulle spese dell'opera. Se infatti ci avessi detto la verità, non ci avresti mai convinto ad affrontare una simile spesa e ora non esisterebbero questo nobile palazzo e questa cattedrale splendida fra

21. Il passo, che in latino fa riferimento a un "sacellum lucidissimum cuius parietes vitrei inter columnas ad medium tenuissimas colligati tenentur" (Piccolomini, 2008, pp. 28-29) è citato in proposito da Alessandro Angelini (2005, p. 23) in un contributo che ripercorre numerose ecfrasi, non solo pientine, dei Commentarii.

22. Henk van Os (1987, pp. 32-38) ha cercato molteplici ragioni per questa soluzione e ne ha riconosciuto la relazione con il tema della luce; un tema che per Mauro Mussolin (2005, p. 245) "sembra più derivare da testi paleocristiani della Chiesa delle origini, che dalle più tarde interpretazioni medievali riconducibili all'abate Suger e all'origine del Gotico" e non corrispondeva per nulla ai gusti di Leon Battista Alberti, che per la venerazione preferiva l'oscurità (ibidem).

23. Piccolomini, 2008, pp. 1764-1765. Per la pala di Giovanni di Paolo datata 1463 e quella di Matteo di Giovanni databile sul 1464, si veda la bibliografia citata supra a nota 20. 
quante sono in Italia. Per il tuo inganno sono stati innalzati questi illustri edifici, che tutti ammirano, con l'eccezione di pochi, morsi dal livore dell'invidia. Noi ti ringraziamo e ti consideriamo meritevole di grande onore fra tutti gli architetti del nostro secolo. (Piccolomini, 2008, pp. 1766-1767).

Tali parole palesano la grandezza di un mecenate, tanto cosciente della propria impresa, da emanare il 16 settembre 1462 una bolla che andava contro ogni consuetudine, vietando qualsiasi intervento successivo nella sua chiesa. Allora gli edifici sacri, anche i più illustri, recavano le più bizzarre e indigeste sedimentazioni, frutto di accozzaglie di dotazioni che la Chiesa usava accogliere guardando più alla cassa, che alle conseguenze estetiche. Pio II, invece ordinò, a pena di anatema, che nel suo tempio

nessuno seppellisca dei morti ad eccezione delle tombe assegnate ai sacerdoti e ai vescovi; nessuno violi il candore delle pareti e delle colonne; nessuno appenda tavole dipinte; nessuno eriga nuove cappelle o nuovi altari; nessuno muti la forma di questa chiesa, sia di quella superiore, sia di quella inferiore. (Piccolomini, 2008, pp. 1768-1769)..$^{24}$

Evidentemente egli considerava insuperabili gli esiti raggiunti, e intendeva eternarli nel tempo, anche se così ovviamente non sarebbe stato. ${ }^{25}$

Resta quindi il racconto di come, muovendo da questi due edifici frutto di un iniziale progetto "privato", Enea Silvio avesse trasformato l'intero villaggio, tramite gli espropri ben pagati, e la realizzazione di nuove costruzioni, in una città nata da una precisa pianificazione sua e del Rossellino. Così il Piccolomini (2008, pp. 1768-1771) volle a sinistra della Cattedrale la casa per il prevosto e i canonici, che potevano comodamente accedere in chiesa da una porta laterale; poco lontano, sul lato di fronte al suo palazzo, egli acquistò la vecchia residenza del pretore e delle magistrature, che affidò al vicecancelliere Rodrigo Borgia, "perché l'abbattesse e costruisse in quel posto il palazzo vescovile, donandolo alla beata Vergine Maria"; lo stesso fece con i vecchi edifici sul lato opposto al Duomo, facendo innalzare al loro posto un palazzo con porticato, per residenza delle magistrature civiche e sede dei consigli, ragione per cui era dotato di un salone, di camere, di un'armeria, di una torre con campana e orologio, e di un carcere. E per tutto questo "ingaggiò egli stesso i muratori e pagò una parte del loro salario, poiché voleva che la piazza fosse circondata da nobili edifici". Segue l'elenco dei membri della corte che, oltre al vicecancelliere, parteciparono all'ambiziosa iniziativa: tra coloro che costruirono palazzi vi furono il cardinale di Arras Jean Jouffroy e il cardinale di Pavia Giacomo Ammannati, il tesoriere e Gregorio Lolli "gettarono le fondamenta", mentre

24. Il testo dei Commentarii altro non riferisce che le prescrizioni contenute in una bolla emanata da Pio II il 16 settembre 1462, trascritta in Mannucci, 1937, p. 31.

25. La bolla del 16 settembre 1462 fu infatti revocata da Gregorio XIII il primo marzo 1585, il quale concesse di modificare l'interno del Duomo, che nel 1600 vide innalzare sull'altare maggiore un monumentale tabernacolo eucaristico corrispondente alle norme del Concilio di Trento, poi smembrato con i ripristini novecenteschi; si veda Martini 2009, pp. 355-361 (anche per ulteriori trasformazioni). 
il cardinale di Mantova Francesco Gonzaga comprò un'area edificabile, e il cubiculario del papa Tommaso del Testa Piccolomini, "i ministri del piombo e parecchi cittadini di Pienza, abbattute le antiche case, ne costruirono di nuove, in modo che in nessun punto la città presentava più l'antico aspetto" (Piccolomini, 2008, pp. 1768-1771). ${ }^{26}$

Ripercorrere Pienza attraverso i Commentarii significa cogliere appieno l'assoluta efficacia di un progetto in cui Pio II seppe mettere insieme la spregiudicatezza politica, un gusto estetico cosmopolita e capace di fare dialogare linguaggi diversi, la ricerca di una razionale e comoda funzionalità degli ambienti, e una personale capacità di relazionarsi con munificenza e savoir-faire con il professionista cui aveva affidato la direzione dei lavori.

Che tutto questo, a distanza di oltre mezzo millennio, rappresenti un'indiscutibile lezione di metodo di pianificazione urbana non dovrebbe essere necessario ricordarlo. E invece merita ancora farlo, soprattutto nel momento in cui l'architettura contemporanea si balocca con l'impianto trapezoidale immaginato dal Rossellino per la Piazza di Pio II, immaginando di allestirvi improbabili "primule" di dubbio gusto, progettate per scongiurare la peste del terzo millennio, ma che, una volta messe in piedi, finirebbero per ammorbare le più belle piazze d'Italia, con effetto analogo a quello di un disco fucsia sparato su una delle celebri Città ideali dipinte (fig. 10-11). ${ }^{27}$

A ciò si aggiunga che la storia che abbiamo riepilogato fin troppo rapidamente è quella di una città concepita, in tutto e per tutto, con grande intelligenza. E il tema dunque è di enorme attualità, poiché l'ultima frontiera della progettazione urbana riguarda proprio una città che, soprattutto tramite le innovazioni tecnologiche, vuole essere intelligente: la cosiddetta "smart city". Cosa sia Pienza lo sappiamo, non solo attraverso i Commentarii, ma potendo ancora sperimentare i suoi spazi urbani, domestici ed ecclesiastici in tutta la loro materialità, e nell'impatto che hanno sui nostri sensi. Cosa sia la "smart city" resta più difficile dirlo, perché le realizzazioni tendono a essere evanescenti, astrattive e molto concettuali, come conseguenza di teorizzazioni non poco generiche, che puntano tutto sulla tecnologia e tendono a non accennare mai a quei valori estetici che nel vivere la dimensione urbana, anche nei rapporti sociali, fanno una decisa differenza. Per esempio, per la Comunità europea:

A smart city is a place where traditional networks and services are made more efficient with the use of digital and telecommunication technologies for the

26. Per i vari palazzi pientini si vedano per tutti: Mack 1987, pp. 104-155; Nevola 2005, 189196; Mack 2012, ricordando che quello di Rodrigo Borgia fu ceduto nel 1468 al vescovo Tommaso del Testa Piccolomini (Chironi 2009, p. 48).

27. Il riferimento è ai padiglioni progettati da Stefano Boeri sul finire del 2020 per la campagna di vaccinazione anti-covid-19 (www.stefanoboeriarchitetti.net), tra i quali non ne mancava uno pensato per essere ambientato nella piazza di Pienza, che illustro accanto una sarcastica immagine della Città ideale del Walters Art Museum di Baltimora guastata da un disco fucsia, messa a corredo del duro e condivisibile giudizio di Fabio Grasso: https:// emergenzacultura.org/2020/12/16/il-covid-e-larchitettura-petalosa/. 
benefit of its inhabitants and business. A smart city goes beyond the use of information and communication technologies (ICT) for better resource use and less emissions. It means smarter urban transport networks, upgraded water supply and waste disposal facilities and more efficient ways to light and heat buildings. It also means a more interactive and responsive city administration, safer public spaces and meeting the needs of an ageing population. (http:// ec.europa.eu/eip/smartcities/).

Di definizioni, tuttavia, se ne possono trovare a centinaia, ne scelgo una per tutte, perché muove dalla domanda "ma che cos'è - concretamente - la smart city?" e risponde - direi ben poco concretamente - come segue:

La smart city è una proiezione astratta di comunità del futuro, un perimetro applicativo e concettuale definito da un insieme di bisogni che trovano risposte in tecnologie, servizi e applicazioni riconducibili a domini diversi: smart building, inclusion, energy, environment, government, living, mobility, education, health, e molto altro ancora. Tali tecnologie, servizi ed applicazioni non costituiscono di per sé né singolarmente né collettivamente una smart city, se non vengono integrate in una piattaforma che assicuri interoperabilità e coordinamento, ma soprattutto la definizione di appropriati strumenti di governance e finanziamento, elementi essenziali alla realizzazione della visione politica e sociale costitutiva della smart city. La smart city è quindi in primo luogo una collezione di problemi rilevanti da affrontare e di idee per risolverli, un insieme di modelli di inclusione, di regole di ingaggio tra sistema pubblico e privato, di nuova strumentazione finanziaria, di innovazione nella Pubblica Amministrazione, di procedure di procurement, di azioni di semplificazione e trasparenza, di regolamentazione, su cui la Pubblica Amministrazione sappia formulare promesse credibili nel medio periodo (Calderini \& Reviglio, 2013).

Si potrebbe continuare all'infinito con quello che farà sempre l'effetto di un ermetico loop di speculazioni, tanto cariche di vocaboli inglesi di tendenza, quanto distanti dalla realtà quotidiana. Speculazioni che, tra l'altro, tendono di norma a ignorare presuntuosamente il problema del patrimonio storico e storicizzato, tanto che per le vestali delle smart city è tranquillamente lecito deturpare un paesaggio urbano per migliorare il segnale del nostro cellulare, o piazzare una rastrelliera per bici elettriche sul cantone di una piazza medievale, perché di norma non si rendono proprio conto di ciò che hanno fatto.

Pur rischiando di banalizzare, è difficile non cogliere la decisiva differenza di approccio tra la generica retorica di questi toni e lo stile chiaro e diretto adottato da Enea Silvio Piccolomini per farci conoscere la sua città. La cultura umanistica, evidentemente, ha ancora molto da insegnare, soprattutto in merito alla praticità, e non solo. Ma su questo meriterà trovare ulteriori occasioni di riflessione. ${ }^{28}$

28. Anche per questo, insieme con Mario Bevilacqua, Elena Svalduz, Andrea Spiriti, Francesco Ceccarelli, Emanuela Ferretti e Maria Beltramini, ci stiamo coordinando per dare vita al progetto di una rete per lo studio delle città ideali del Rinascimento, che presto dovrebbe iniziare a operare. 


\section{Bibliografia}

Adams, N. (1985). The Acquisition of Pienza 1459-1464. Journal of the Society of Architectural Historians, 44, 2, 99-110.

Adams, N. (1998). Pienza. In Fiore, F.P. (Ed.). (1998). Storia dell'architettura italiana. Il Quattrocento (pp. 314-329). Milano: Electa.

Angelini, A. (Ed.). (2005). Pio II e le arti. La riscoperta dell'antico da Federighi a Michelangelo. Siena - Cinisello Balsamo: Monte dei Paschi di Siena - Silvana editoriale.

Angelini, A. (2005). Templi di marmo e tavole quadre. Pio II e le arti nei Commentarii. In Angelini 2005, pp. 18-43.

Angelini, A. (2011-2012). Le tavole con la "città ideale" a confronto. In margine alla mostra di Urbino: proposte per autori e significati. 1492, 4-5, 65-87.

Barbacci, A. (1934). Il restauro del Duomo di Pienza. La Diana, IX, 5-135.

Bellosi, L. (1990). Giovanni di Francesco e l'arte fiorentina di metà Quattrocento. In Bellosi, L. (Ed.). (1990). Pittura di luce. Giovanni di Francesco e l'arte fiorentina di metà Quattrocento. Catalogo della mostra di Firenze (16 maggio - 20 agosto 1990) (pp. 11-45). Milano: Olivetti.

Bevilacqua, M. (2016). Mura di luce, facciate di diamanti. Metafore del bianco nell'architettura del Quattrocento. Opus incertum, II (nuova serie), 34-47.

Bulian, G., Croci, G., Giorgianni, G., \& Russo, C. (2006). Dati storici, monitoraggio, indagini. In Giorgianni, G. (2006), Pio II, la città, le arti. La rifondazione umanistica dell'architettura e del paesaggio. Catalogo della mostra di Pienza (28 maggio - 8 ottobre 2006). Siena: Protagon.

Burckhardt, J. (1876). La civiltà del secolo del Rinascimento in Italia, 2 voll. Firenze: Sansoni.

Calderini, M, \& Reviglio, E. (2013). Premessa. In Reviglio E., Camerano S. et al. (2013). Smart City Progetti di sviluppo e strumenti di finanziamento. Roma: Cassa depositi e prestiti.

Carli, E. (1966). Pienza. La città di Pio II. Roma: Editalia.

Chironi, G. (Ed.). (2000). L’archivio diocesano di Pienza. Siena: Amministrazione provinciale di Siena.

Chironi, G. (2009). Un mondo perfetto. Istituzioni e societas christiana nella Pienza di Pio II. In Nevola 2009, pp. 39-50.

De Brosses, C. (1973). Viaggio in Italia. Lettere familiari. Bari: Laterza.

Fattorini, G. (2006). Vecchietta "pittore di luce". La pala di Spedaletto. In Detti, T. (Ed.) (2006). La terra dei musei: paesaggio arte storia del territorio senese (pp. 400405). Siena - Firenze: Monte dei Paschi di Siena - Giunti.

Mack, C.R. (1987). Pienza. The Creation of a Renaissance City. Ithaca-London: Cornell University Press.

Mack C.R. (2012). Beyond the Monumental: the Semiotics of Papal Authority in Renaissance Pienza. Southeastern College Art Conference Review, 16,2, 124-150.

Mannucci, G.B. (1937). Pienza: arte e storia. Siena: Tipografia San Bernardino (terza edizione ampliata e corretta, che segue quelle del 1915 e 1927).

Martini, L. (2005). Tabulae pictae e altri ornamenti per la Cattedrale di Pienza. In Angelini 2005, pp. 250-279.

Martini, L., Santi B. (2006). Il Palazzo Piccolomini di Pienza: guida al palazzo e alle sue collezione. Siena: Cantagalli.

Martini, L. (2009). Le pale d'altare nel duomo di Pienza. In Nevola 2009, pp. 354-376. 
Mussolin, M. (2005). "Cathedralis effecta est: il Duomo di Pienza e il rinascimento cristiano di Pio II. In Angelini 2005, pp. 214-249.

Nevola, F. (2005). Architettura civile. In Angelini 2005, pp. 182-213.

Nevola F. (Ed.). 2009. Pio II Piccolomini: il papa del Rinascimento a Siena. Atti del convegno internazionale di studi di Siena (5-7 maggio 2006). Siena: Protagon.

Paardekooper, L. (2009). La diffusione della tavola quadrata nel senese: un influsso pientino o meno? In Nevola 2009, pp. 377-409.

Palladino P. (1994). "Pittura in una casa di vetro": un riesame e una proposta sul programma decorativo di Pio Il per la cattedrale di Pienza. Prospettiva, 75/76, 100-108.

Pellegrini, M. (2000). Pio II. In Enciclopedia dei papi (Vol. 2, pp. 663-685). Roma: Istituto della enciclopedia italiana fondato da Giovanni Treccani.

Pellegrini, M. (2015). Pio II, papa. In Dizionario biografico degli Italiani (Vol. 83, pp. 794-803). Roma: Istituto della enciclopedia italiana fondato da Giovanni Treccani.

Piccolomini, E.S., papa Pio II (2008). I commentarii (a cura di L. Totaro). Milano: Adelphi (nuova edizione ampliata, rispetto all'originale del 1984).

Pieper, J. (2000). Pienza. Il progetto di una visione umanistica del mondo. StuttgartLondon: Axel Menges.

Spesso, M. (1997). Wiener Neustadt: un riferimento per Pienza. Commentari d'arte, II, 4, 16-24.

Spesso, M. (2018). Premesse a Pienza: architettura e umanesimo integrale. Milano: Franco Angeli.

Spiriti, A. (2018). Castiglione Olona. La prima città ideale dell'umanesimo. Milano: Mimesis. Tönnesmann, A. (1990). Pienza Städtebau und Humanismus. München: Hirmer.

Van Os (1987). Painting in a House of Glass: The Altarpieces of Pienza. Simiolus, 17, 23-38.

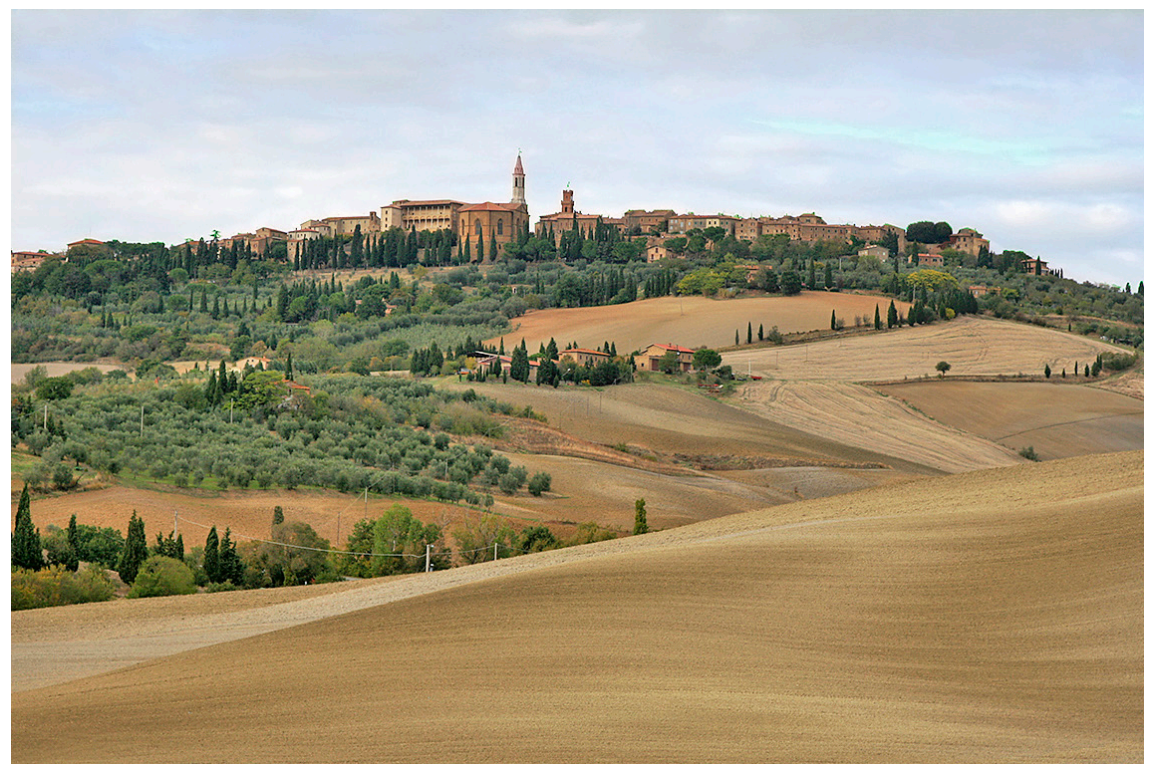

1. Pienza vista in lontanza dalla Val d'Orcia. 


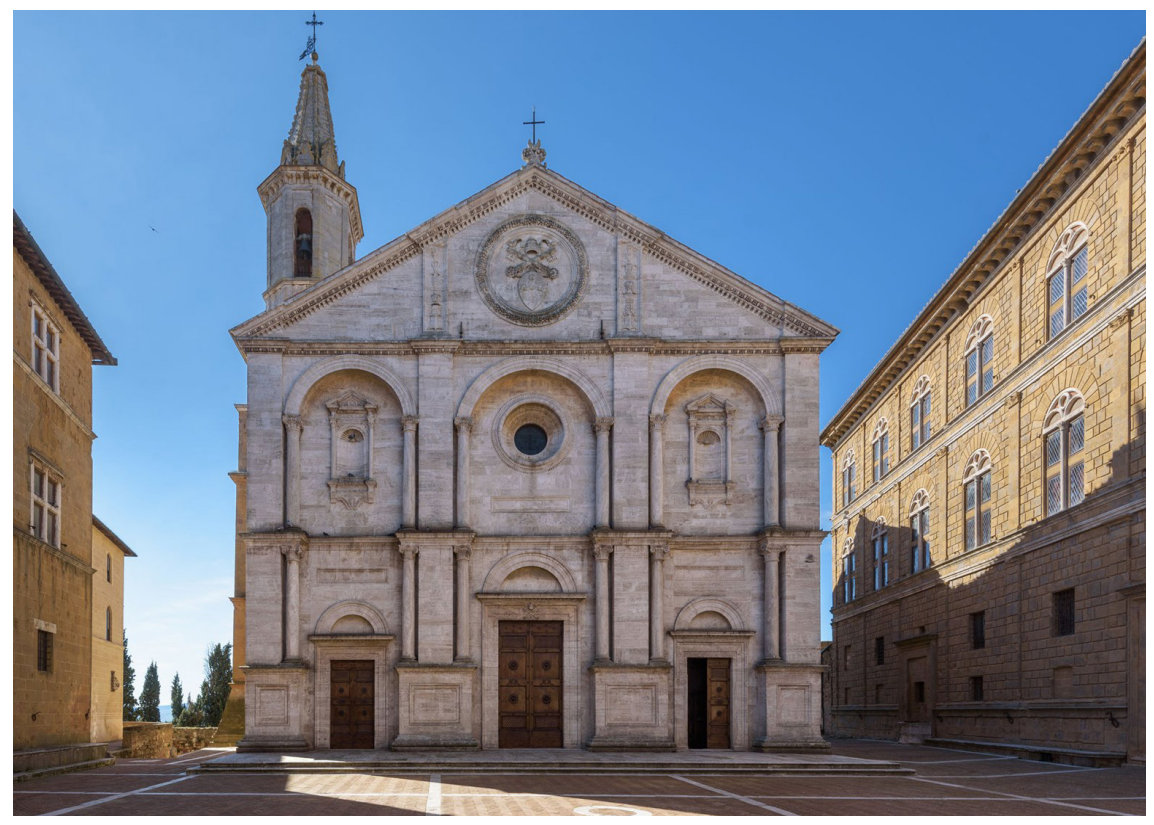

2. Pienza: Piazza Pio II.

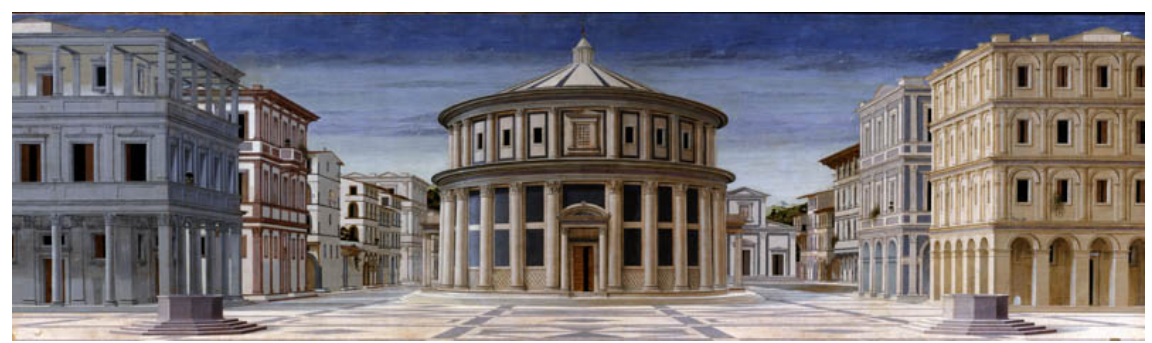

3. Pittore centroitaliano (Donato Bramante?): Prospettiva urbana detta Città ideale. Urbino, Galleria Nazionale delle Marche. 


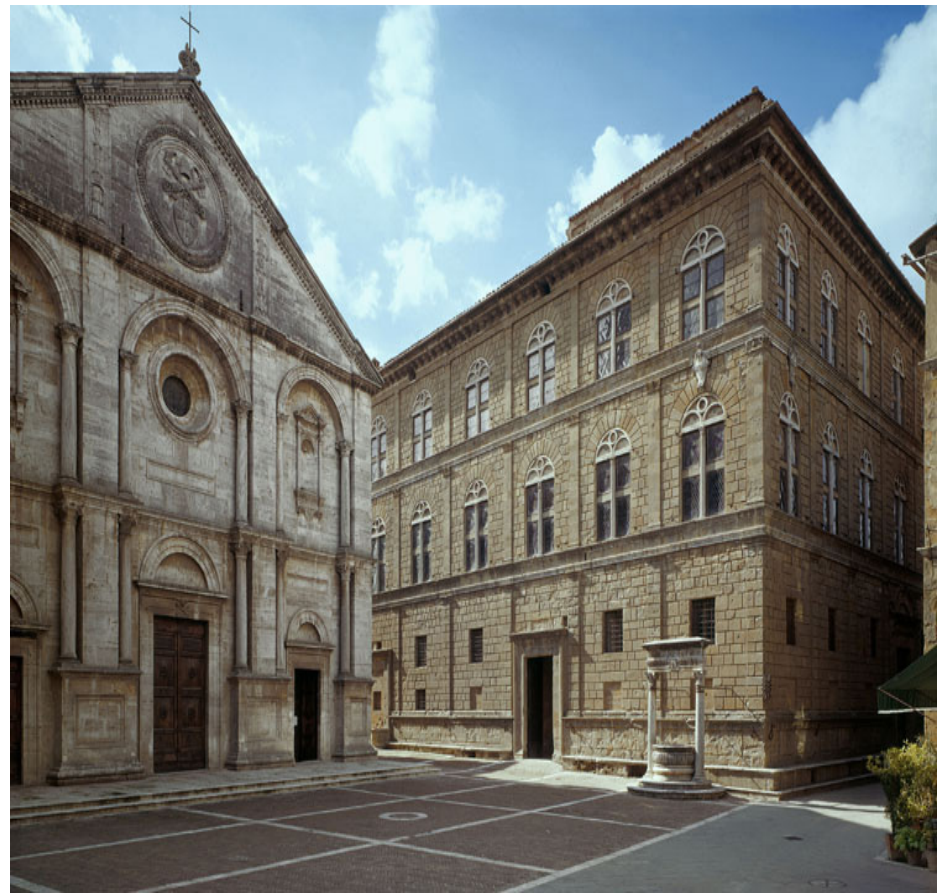

4. Pienza: Palazzo Piccolomini.

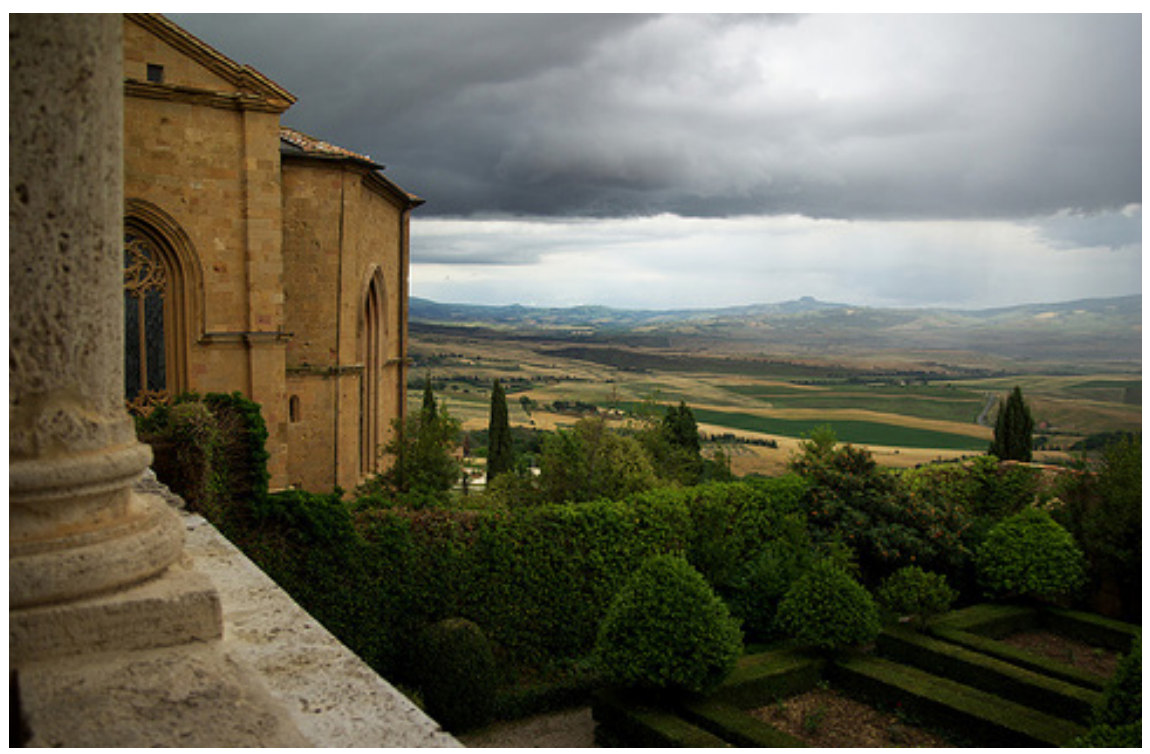

5. Pienza: veduta della Val d'Orcia dalla loggia di Palazzo Piccolomini. 


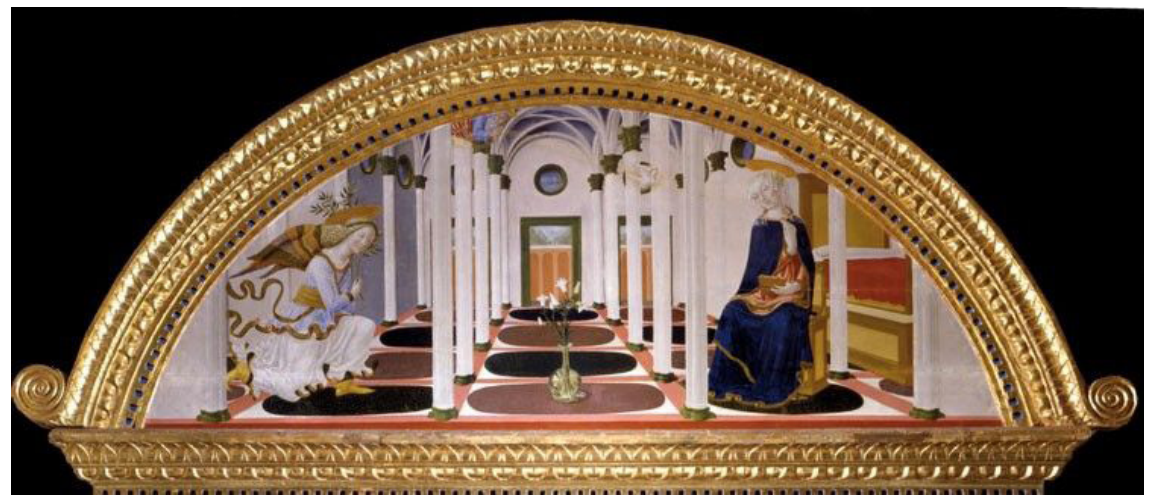

6. Lorenzo di Pietro detto il Vecchietta: Annunciazione (particolare della Pala di Spedaletto). Pienza, Museo Diocesano di Palazzo Borgia.

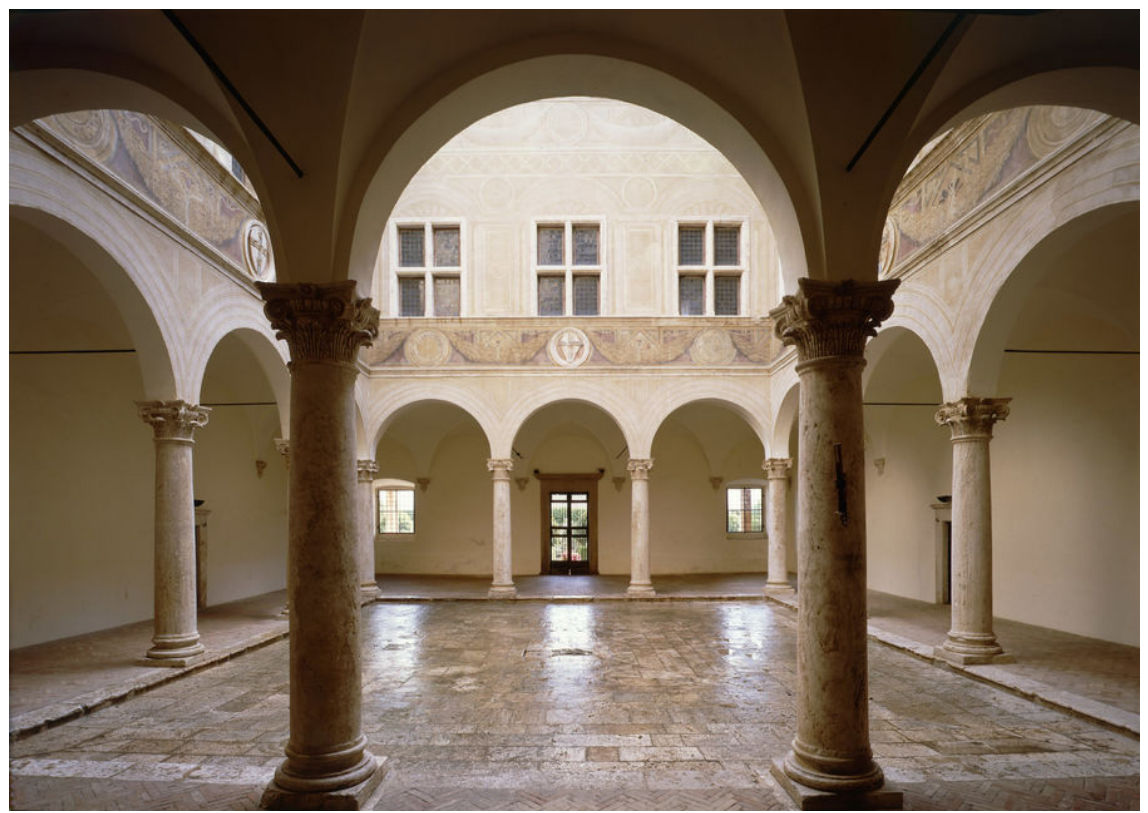

7. Pienza: peristilio del Palazzo Piccolomini (con il giardino sul fondo) 


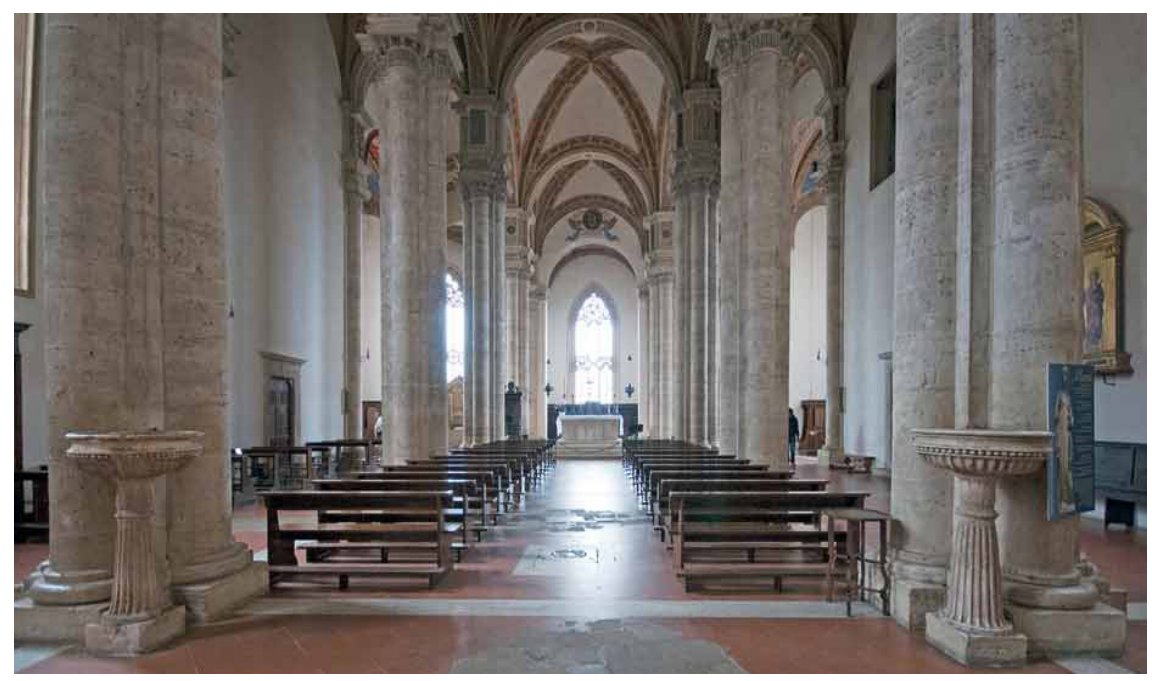

8. Pienza: interno della Cattedrale.

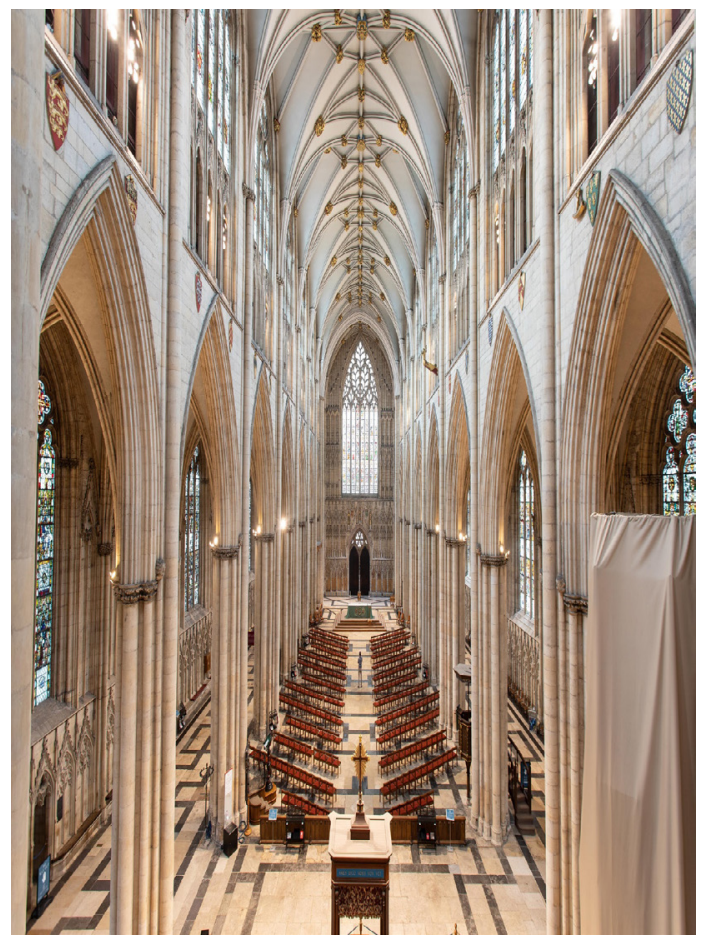

9. York: interno della Cattedrale. 


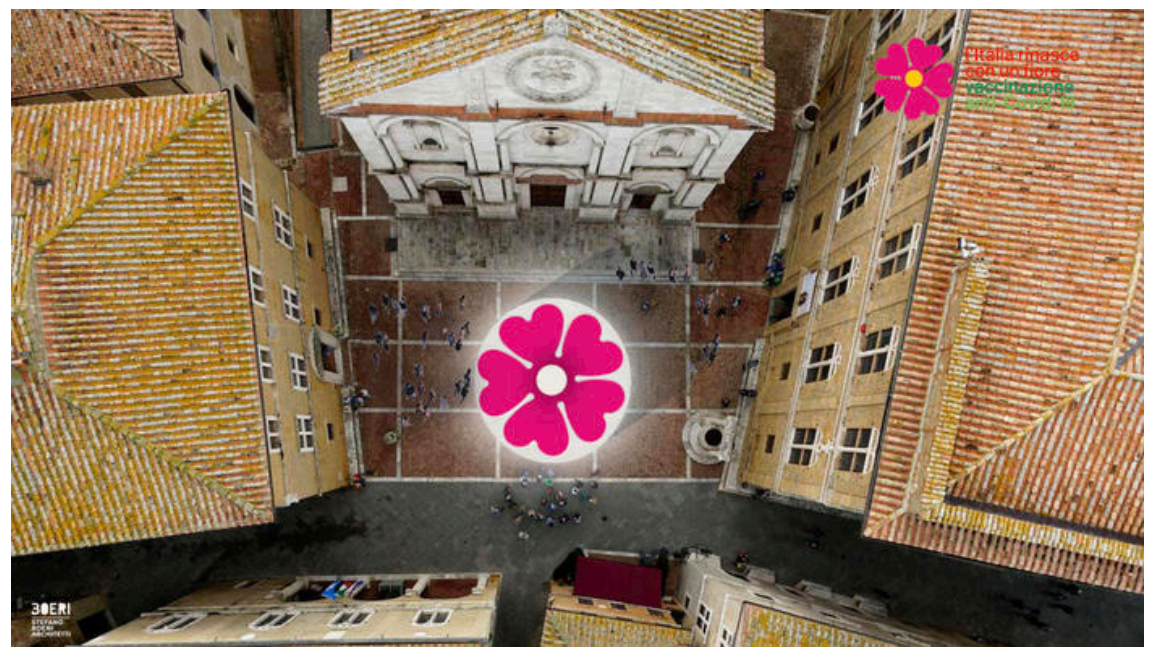

10. Stefano Boeri Architetti: campagna di vaccinazione anti-covid -19 (dicembre 2020).

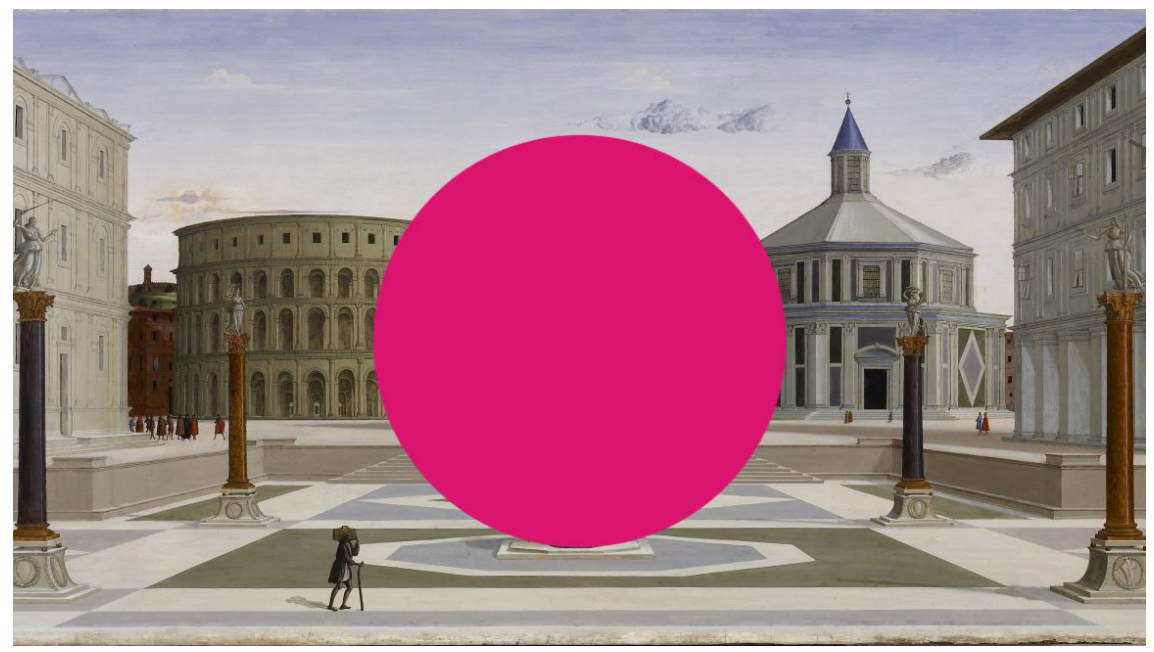

11. Punto rosso in piazza rinascimentale: da emergenzacultura.org (16 dicembre 2020). 